\title{
Manutenção das classes de equivalência e transferência de função: uma investigação por meio de escolhas alimentares de crianças ${ }^{*}$ \\ Maintenance of Equivalence Classes and Transfer of Functions: an investigation through the children's food choice
}

\section{Manutención de las clases de equivalencia y transferencia de función: una investigación mediante la elección de alimentos por niños}

\section{Silvana Lopes dos Santos ${ }^{1}$, Julio Cesar Coelho de Rose ${ }^{2}$}

\footnotetext{
[1] [2] Universidade Federal de São Carlos I Título abreviado: Manutenção da transferência de função e escolhas alimentares | Endereço para correspondência: Universidade Federal de São Carlos, Departamento de Psicologia, Laboratório de Estudos do Comportamento Humano (LECH). Rodovia Washington Luis, km 235, Caixa Postal: 676, São Carlos, SP, Brasil; CEP 13565-905 I Email: silvana@ufscar.br I DOI: 10.18761/pac.2016.022
}

\begin{abstract}
Resumo: Esta pesquisa investigou em que medida a equivalência entre personagens e marcas pode influenciar a escolha por alimentos. Também buscou-se verificar a estabilidade da formação de classes e transferência de função. 11 crianças pré-escolares foram submetidas a um procedimento de emparelhamento com o modelo para a formação de duas classes de equivalência (A1B1C1 e A2B2C2) na qual uma continha um personagem de que ela gostava (A1) e outro de que ela não gostava (A2). Os demais estímulos eram figuras geométricas (B1 e B2) e símbolos abstratos ( $\mathrm{C} 1$ e C2). Após a formação de classes, foram conduzidos três testes de escolha e preferência que consistiam em oferecer aos participantes dois alimentos idênticos em embalagens com rótulos diferentes. No Teste 1, os rótulos eram $\mathrm{C} 1$ e $\mathrm{C} 2$; No Teste 2, C2 e um símbolo novo; e no Teste 3, C1 e um outro símbolo novo. Após duas semanas, estes testes, assim como os de formação de classes de equivalência, foram repetidos. A maioria das crianças escolheu e demonstrou preferência pelo alimento com o símbolo equivalente ao personagem de que ela gostava (C1). Os Testes 2 e 3 foram inconclusivos. Os testes de manutenção indicaram estabilidade na formação das classes e transferência de função. Conclui-se que a equivalência de estímulos é um paradigma comportamental útil para investigação de atitudes e preferências por produtos.
\end{abstract}

Palavras-chave: equivalência de estímulos, transferência de função, manutenção das classes de equivalência, escolhas e preferência alimentares, personagem, crianças.

\footnotetext{
${ }^{\star}$ Este trabalho foi parte do programa científico do Instituto Nacional de Ciência e Tecnologia sobre Comportamento, Cognição e Ensino (INCT-ECCE), financiado pelo CNPq (Processo 573972/2008-7) e FAPESP (Processo 2008/57705-8) e coordenado por Deisy de Souza. Agradecemos à coordenadora do INCT-ECCE pelo apoio a esta pesquisa.
} 
Abstract: This study investigated to what extent equivalence between cartoon character and brand can influence food choice. Also sought to verify the stability of formation of classes and the transfer of function. Eleven Preschool children formed two equivalence classes (A1B1C1 and $\mathrm{A} 2 \mathrm{~B} 2 \mathrm{C} 2)$ in which one class had a liked character (A1) and the other a disliked character (A2). The other stimuli were geometric figures (B1 and B2) and abstract symbols ( 1 1 and C2). Three preference tests were conducted where the children had to choose from two samples of the same snack, differing only by the label on the packaging. In Test 1 the labels were symbols C1 and C2; in Test 2, C2 and a new symbol; and in Test 3, C1 and the other new symbol. After two weeks were repeated these tests and the equivalence test. Most children chose first, and reported to like more, the snack labeled with the symbol equivalent to the liked character. The Tests 2 and 3 were inconclusive. The maintenance tests demonstrated the formation of equivalence classes and transfers of functions were stable. It has concluded that stimulus equivalence is a useful paradigm to a behavioral investigation of attitudes and preferences for products.

Keywords: stimulus equivalence, transfer of function, Maintenance of equivalence classes, food choice and preference; cartoon character, children.

Resumen: Esta investigación estudió en qué medida la equivalencia entre personajes y marcas puede influenciar la elección de alimentos. También se buscó verificar la estabilidad de la formación de clases y transferencia de función. 11 niños prescolares formaron dos clases de equivalencia (A1B1C1 E A2B2C2) en que una contenía un personaje que le gustaba (A1) y otro que no le gustaba (A2). Los demás estímulos eran figuras geométricas (B1 y B2) y símbolos abstractos ( $\mathrm{C} 1$ y C2). Después, fueron conducidos tres testes de elección y preferencia que consistían en ofrecer a los participantes dos alimentos idénticos en embalajes con rótulos diferentes. En el teste1, los rótulos eran $\mathrm{C} 1$ y C2, en el teste 2, $\mathrm{C} 2$ y un símbolo nuevo; y en el test 3, C1 y otro símbolo nuevo. Después de dos semanas estos testes, así como los de equivalencia, fueron repetidos. La mayoría de los niños escogió y demostró preferencia por el alimento con el símbolo equivalente al personaje que le gustaba (C1). Los testes 2 y 3 no fueron conclusivos. Los testes de manutención indicaron estabilidad en la formación de clases y transferencia de función. Se concluye que la equivalencia de estímulos es un paradigma conductual útil para la investigación de actitudes y preferencias por productos.

Palabras-clave: equivalencia de estímulos, transferencia de función, manutención de las clases de equivalencia, elecciones y preferencias alimentares, personaje, niños. 
O paradigma de equivalência de estímulos tem sido uma abordagem que possibilita a investigação de como estímulos distintos entre si se tornam substituíveis em diversos contextos (Sidman, 1994; Sidman, 2000, Sidman \& Tailby, 1982). Várias pesquisas têm demonstrado que se as relações entre os estímulos da classe são de equivalência, a atribuição de uma função a um membro desta classe é transferida para os demais (e.g., Amd, Barnes-Holmes, \& Ivanoff, 2013; Barnes-Holmes, Barnes-Holmes, Smeets, Luciano, 2004; de Rose, Mcllvane, Dube, Galpin, \& Stoddard, 1988; Dougher, Augustson, Markham, Greenway, \& Wulfert, 1994; Wulfert \& Hayes, 1988). Dougher et al. (1994), por exemplo, pareou um membro de uma classe (CS) com choque elétrico leve (US) que eliciou uma resposta galvânica da pele (GSR). O CS adquiriu a função de eliciar uma GSR, como esperado pelo condicionamento clássico. No entanto, outros membros da classe, embora nunca tivessem sido pareados com o choque, também passaram a eliciar a mesma resposta.

Fenômeno semelhante também é observado quando símbolos abstratos se tornam equivalentes a estímulos que já possuem um significado pré-experimental. Pesquisas desenvolvidas por Bortoloti e de Rose $(2007,2009,2012)$ têm constatado que classes formadas por estímulos significativos, como expressões faciais de emoções (ex. raiva, alegria e neutralidade), e símbolos abstratos, tendem a fazer com que tais símbolos sejam avaliados de forma semelhantes às faces, indicando a transferência de funções entre eles. Diferentes medidas independentes têm possibilitado a validação de pesquisas nesta área, tais como o diferencial semântico (Bortoloti \& de Rose, 2007, 2009, 2012; de Almeida \& de Rose, 2015), o Implicit Relational Assessment Procedure (IRAP; Bortoloti \& de Rose, 2012), e medidas de potencias relacionados a eventos (PREs) (BarnesHolmes et al., 2005; Bortoloti, Pimentel, \& de Rose, 2014; Haimson, Wilkinson, Rosenquist, Ouimet, \& McIlvane, 2009).

Partindo do princípio de que estímulos neutros podem ser avaliados de acordo com a classe a que pertencem, a equivalência de estímulos tem sido uma metodologia útil de investigação para a área de formação e mudança de atitudes a partir de uma abordagem analítica comportamental (de Carvalho, \& de Rose, 2014; Grey, \& Barnes,
1996; Haydu, Camargo, \& Bayer, 2015; Mizael, de Almeida, Silveira, \& de Rose (2016); Mizael. Santos, \& de Rose (2016); Moxon, Keenan, \& Hine, 1993; Watt, Keenan, Barnes, \& Cairns, 1991). Grey e Barnes (1996, Estudo 1) foram pioneiros na investigação da formação de atitudes com o uso deste paradigma. Eles treinaram universitários a formarem três classes com sílabas sem sentido (A1B1C1, A2B2C2 e A3B3C3) utilizando o Matching to Sample (MTS). Na sequência, cada participante assistia a dois filmes: um com conteúdo sexual e o outro com cenas religiosas. Cada vídeo possuía um rótulo na capa com uma sílaba sem sentido (B1 e B2) das classes formadas no MTS. Os participantes classificaram estes vídeos como "bons" ou "ruins" e, em seguida, tiveram que fazer a mesma coisa com outros quatro vídeos não vistos, rotulados com as sílabas previamente utilizadas durante a fase de treino (A1, C1, A2 e C2). Os resultados mostraram que os participantes classificaram os vídeos não vistos de acordo com a classe a que pertenciam os rótulos, por exemplo, os filmes com os rótulos A1 e $\mathrm{C} 1$ eram avaliados da mesma forma que B1. Este estudo demonstrou, portanto, a formação de atitudes sem a necessidade de uma história direta, ou seja, mesmo não assistindo aos vídeos, houve uma avaliação positiva ou negativa a depender da classe a que pertenciam os rótulos dos filmes.

Seguindo esta mesma abordagem, alguns trabalhos têm investigado se preferências ou atitudes em relação a marcas e produtos podem ser influenciadas pelas relações de equivalência (Arntzen, Fagerstrom, \& Foxall, 2016; BarnesHolmes, Keane, Barnes-Holmes, \& Smeets, 2000; Santos, \& de Rose, no prelo; Smeets, \& BarnesHolmes, 2003; Straatmann, Almeida, \& de Rose, 2014). Uma destas pesquisas foi a de Smeets e Barnes-Holmes (2003), que testaram se a transferência de função entre estímulos poderia influenciar a preferência de crianças por refrigerantes. Eles conduziram um treino de MTS para estabelecer duas classes de estímulos: uma delas contendo a figura de um personagem infantil (Ernie) e a outra contendo a figura de uma criança chorando. Os demais estímulos eram figuras geométricas e símbolos abstratos. Após a formação das classes, eram apresentadas duas garrafas iguais do mesmo refrigerante. Em uma delas, havia no rótulo 
o símbolo da classe do personagem infantil, e na outra, o símbolo da classe da criança chorando. A maioria das crianças escolheu provar primeiro, e demonstrou preferência, pelo refrigerante cujo rótulo pertencia à classe da figura preferida. Tais resultados foram condizentes aos encontrados em estudo anterior com estudantes universitários, utilizando palavras emotivas ao invés de figuras (Barnes-Holmes et al., 2000).

Pesquisa de Santos e de Rose (no prelo) replicou estes resultados com algumas variações no procedimento, entre elas o uso do matching atrasado (Bortoloti, \& de Rose, 2007, 2009, 2012). O objetivo foi investigar a transferência de função por meio de testes adicionais. Crianças pré-escolares formaram duas classes compostas por três membros (A1B1C1 e A2B2C2). O estímulo A1 era a figura de um personagem preferido ("positivo") e o A2 era um personagem do qual a criança não gostava ("negativo"), de acordo com sua própria escolha. Após esta fase, os participantes foram submetidos a três testes de escolha e preferência com dois alimentos iguais, somente com os rótulos diferentes: um com o símbolo equivalente ao personagem "positivo" (C1) e o outro ao personagem "negativo" (C2); outro com o símbolo equivalente ao personagem "negativo" (C2) e um símbolo novo; e o terceiro com o símbolo equivalente ao personagem "positivo" $(\mathrm{C} 1)$ e uma marca de alimento reconhecida pela criança. Os resultados do teste 1, como nos estudos anteriores, demostraram que a maioria das crianças escolheu e preferiu o alimento com o símbolo equivalente ao personagem preferido. No segundo teste, houve maior escolha pelo alimento com o símbolo novo no rótulo ao invés do outro com o símbolo da classe do personagem que a criança não gostava. $\mathrm{O}$ terceiro teste foi inconclusivo. A princípio, tais dados parecem indicar tanto a transferência de função positiva quanto negativa. No primeiro caso, refere-se à escolha do alimento com símbolo equivalente ao personagem de que a criança gostava em comparação ao que não gostava (Teste 1) e, no segundo caso, pela suposta rejeição do alimento equivalente ao personagem de que a criança não gostava, inferida a partir da escolha do símbolo novo (Teste 2). No entanto, somente a transferência de função negativa daria conta desta explicação na medida em que poderia levar aos mesmos resultados, ou seja, a escolha do símbolo "posi- tivo" no teste 1 e do símbolo novo no teste 2 , ambas por rejeição ao "negativo".

O propósito do presente estudo foi estender os achados de Santos e de Rose (no prelo). Um dos objetivos foi verificar tanto a manutenção das classes de equivalência como a transferência de função, tendo em vista haver poucos estudos nesta área (Rehfeldt, \& Dymond, 2005; Silveira et al., 2016). Uma das hipóteses é que haveria a transferência de função demonstrada pelos testes de preferência e esta se manteria após um período de tempo. Outro objetivo foi investigar a transferência de função tanto positiva quanto negativa. Para tanto, além do Teste 2 utilizado na pesquisa anterior, um terceiro teste foi inserido e consistia na escolha entre um alimento com rótulo contendo o símbolo equivalente ao personagem "positivo" e o outro com um símbolo novo. A hipótese é que o participante escolheria o alimento com o símbolo novo no segundo teste ao invés do alimento com o símbolo da classe do personagem não atrativo, indicando transferência de função negativa. No terceiro teste, a escolha seria pelo alimento com o símbolo equivalente ao personagem positivo e não o símbolo novo, indicando assim a transferência de função positiva. Também se buscou controlar a possível variável "preferência pelo símbolo do rótulo", alternando os estímulos $\mathrm{C} 1$ e C2 entre as classes, ou seja, o mesmo símbolo tinha função "positiva" para alguns participantes e função "negativa" para outros.

\section{Método}

\section{Participantes}

Participaram desta pesquisa 11 crianças pré-escolares com idade entre 5 e 6 anos, sendo sete meninos e quatro meninas, matriculadas em uma Escola de Educação Infantil. Os pais aprovaram a participação da criança, assinando um Termo de Consentimento Livre e Esclarecido com as informações sobre a pesquisa. Eles também tinham que preencher um questionário sobre os hábitos e restrições alimentares de seus filhos. Esta pesquisa foi aprovada pelo Comitê de Ética em Pequisas com Humanos da Universidade Federal de São Carlos (CAAE: 09966612.1.0000.5504). 


\section{Local e materiais}

A coleta de dados foi conduzida em uma sala na própria escola, onde havia uma mesa e 2 cadeiras pequenas infantis, sendo as mesmas normalmente utilizadas pelas crianças. Foi utilizado um computador contendo o Programa MESTRE (Elias, \& Goyos, 2010) para aplicação e registro das sessões. Além deste computador, em cima da mesa de aplicação havia um recipiente de vidro onde eram colocadas bolinhas de gude, após cada acerto na atividade. Ao final da sessão eram oferecidos adesivos de personagens em troca dos pontos obtidos. Utilizou-se também uma filmadora digital para a filmagem das sessões.

$\mathrm{Na}$ fase dos testes de escolha e preferência alimentar, fazia-se uso de embalagens transparentes que continham pequenas amostras de alimentos escolhidos conforme as preferências ou restrições alimentares relatadas no questionário. De maneira geral, os alimentos adotados para a maioria dos participantes foram: bolacha salgada, suco de soja sabor uva e chocolate.

\section{Estímulos}

No treino MTS foram planejadas a formação de duas classes de equivalência com três membros (A1B1C1 e A2B2C2) conforme ilustra a Figura 1. O A1 era a imagem de um personagem de que a criança gostava e o A2 a de um personagem de que ela não gostava. Os estímulos B1 e B2 eram figuras geométricas (quadrado e triângulo, respectivamente). Os estímulos $\mathrm{C} 1$ e C2 eram símbolos abstratos. A Figura 2 também ilustra os dois símbolos abstratos novos usados nos testes de escolha e preferência alimentar.

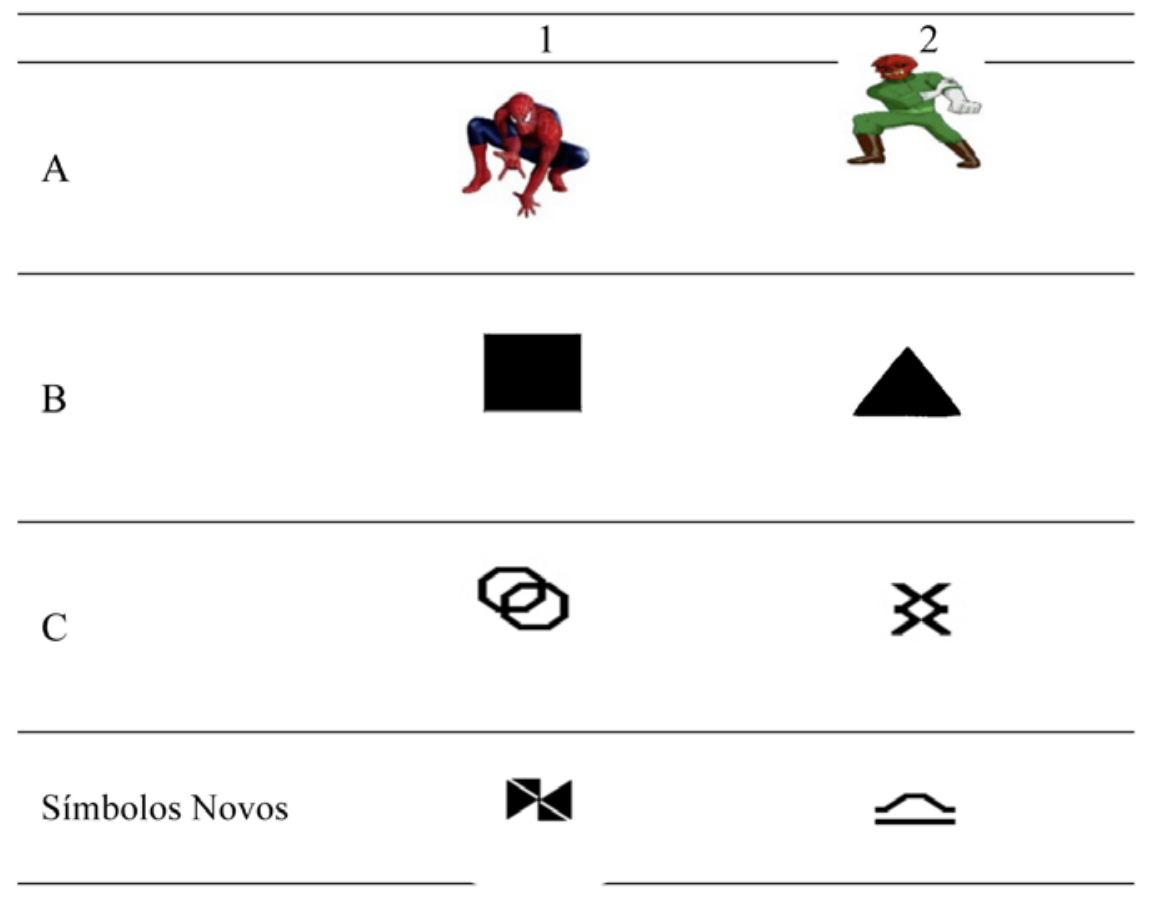

Figura 1. Exemplos de estímulos utilizados no MTS e os símbolos novos dos testes de escolha e preferência alimentar (SN1 e SN2 respectivamente)

\section{Procedimento}

O procedimento foi dividido em quatro fases: "pré-treino", "treino e teste relacional", "testes de escolha e preferência" e "teste de manutenção". As sessões ocorreram em média, duas vezes por semana, sendo seis sessões no total, cuja duração máxima era de 10 minutos cada uma.

\section{Fase 1 - Pré-treino}

O pré-treino ocorreu na primeira sessão e consistiu na escolha dos estímulos A1 (personagem de que a criança gostava) e A2 (personagem que a criança não gostava) e na introdução ao MTS. 
Escolha dos personagens - a escolha dos personagens teve como base a avaliação de preferência pareada (Fisher et al., 1992), que consiste na apresentação simultânea de dois estímulos de uma lista, até que todas as possíveis combinações de pares sejam apresentadas. Neste estudo, a escolha das figuras foi conduzida com a utilização do "jogo da escolha" (Escobal, Elias, \& Goyos, 2012) que tem possibilitado a avaliação de preferência pareada com o uso do computador. Este jogo foi validado tanto para crianças com desenvolvimento típico quanto atípico (Escobal, Elias, \& Goyos, 2014).

Primeiramente, foram selecionados cinco personagens infantis de que as crianças gostavam e cinco de que não gostavam, tendo como base as respostas dos pais ao questionário, bem como as preferências da faixa etária estudada. Ao chegar à sala de coleta, a pesquisadora dizia à criança que ela brincaria com um joguinho no computador, mas antes, gostaria de saber quais os personagens de que ela mais gostava. Era dada a instrução "vão aparecer sempre dois personagens na tela. Eu quero que você aponte qual dos dois você mais gosta". Na tela do computador apareciam sempre duas figuras de personagens de que ela gostava simultaneamente com todas as possíveis combinações, somando 20 apresentações. O personagem com maior número de escolhas era selecionado para o experimento. Se houvesse empate, os personagens eram apresentados pareados novamente, solicitando à criança escolher o preferido. O mesmo procedimento foi adotado para a escolha do personagem de que a criança não gostava. Neste caso a instrução era: "vão aparecer sempre dois personagens na tela. Eu quero que você aponte qual dos dois você menos gosta".

Introdução ao MTS - um treino de MTS foi conduzido para familiarizar os participantes com o método. Este treino consistiu em blocos de 8 tentativas de matching de identidade (escolha do estímulo comparação idêntico à amostra/modelo) utilizando estímulos familiares para a criança (abajur, bola, animais etc.). O procedimento foi semelhante ao que seria conduzido no treino. Eles recebiam a instrução: "Nós vamos brincar de um joguinho agora. Vai aparecer uma figura no centro da tela. Quando você clicar nesta figura, duas outras vão aparecer embaixo. Em uma delas, quando você clicar, ganhará pontos e na outra não". A pesquisadora permanecia ao lado da criança e reforçava seus acertos com expressões verbais (“Isto!", “Muito bem!", "Você entendeu!").

Quando a criança acertava, era exibida na tela uma animação de um porquinho recebendo moeda e, então, era colocada uma bolinha de gude num recipiente de vidro que ficava ao lado do computador. Caso ela errasse, a tela ficava escura por 3 segundos. O critério para participar do estudo era que a criança acertasse $100 \%$ das tentativas. Caso não atingisse o critério, o bloco era repetido até que conseguisse. Todas as crianças atingiram este critério até no máximo dois blocos.

\section{Fase 2 - Treino e teste relacional}

O treino e teste MTS ocorreu nas sessões de 2 a 4 . Adotou-se neste experimento o protocolo Simples para o Complexo (STC, Adams, Fields \& Vehave, 1993), no qual o treino de uma nova relação somente ocorre após o teste de simetria da relação ensinada anteriormente. Como forma de controlar a possível preferência por um símbolo específico, o símbolo que pertencia à classe do personagem "positivo" para alguns participantes, era da classe do personagem "negativo" para os demais.

A sessão era iniciada com um bloco de 12 tentativas de treino das relações $\mathrm{AB}$ (6 A1B1 e 6 A2B2) com matching atrasado (DMTS $1 \mathrm{~s}$ ), sendo que as duas primeiras tentativas continham uma dica da resposta correta (um dedo indicador apontava o estímulo correspondente na tela). A partir do segundo bloco eram 10 tentativas sem dicas (5 A1B1 e 5 A2B2). A seguinte instrução era dada:

Nós vamos brincar agora de um joguinho parecido com o que fizemos no outro dia. Vai aparecer uma figura no centro da tela, você irá clicar e aparecerão duas embaixo. Em uma delas quando você clicar aparecerá o porquinho na tela, você ganhará pontos e eu colocarei uma bolinha de gude aqui neste vidro. Preste bastante atenção pois nas duas primeiras tentativas vai ter um dedo apontando em qual clicar. Você poderá trocar suas bolinhas de gude por um brinde no final.

O participante clicava com o mouse no estímulo (A1 ou A2) no centro superior da tela, ele desa- 
parecia e um segundo depois dois novos estímulos (B1 e B2) surgiam na parte inferior, em posições randomizadas. As consequências para as respostas corretas e incorretas eram as mesmas do pré-treino descritas anteriormente. $\mathrm{O}$ critério a ser atingido para a próxima fase era de, no máximo, um erro no bloco de tentativas. Caso o participante não atingisse este objetivo, o bloco era repetido voltando à linha de base (sem dicas).

$\mathrm{O}$ teste das relações de simetria BA era conduzido com blocos de 8 tentativas (4 B1A1 e 4 B2A2) randomizadas, sem consequências diferenciais. Era dada a instrução: "Agora não vai mais aparecer o porquinho e não vou lhe dizer se você acertou ou errou. Preste bastante atenção e faça o melhor." O critério para a próxima fase era de, no máximo, uma resposta incorreta. Se o critério não fosse atingido até dois blocos de teste, o participante seria excluído do experimento, mas isto não ocorreu para nenhum deles.

$\mathrm{O}$ mesmo procedimento foi adotado para o treino e teste das relações $\mathrm{BC}$ na terceira sessão. $\mathrm{O}$ teste de equivalência era conduzido na quarta sessão onde se testava a transitividade (AC) e a combinação de transitividade e simetria (CA). Foram 8 tentativas $\mathrm{AC}$, seguidas de 8 tentativas de CA randomizadas. A instrução dada ao participante foi a mesma utilizada nos testes de simetria detalhados anteriormente.

\section{Fase 3 - Testes de escolha e preferência alimentar} Testes de escolha e preferência alimentar foram conduzidos para verificar a transferência de função. Esta sessão ocorria na sequência do teste de equivalência ou no dia posterior. Na sessão anterior aos testes era perguntado à criança se ela gostava dos alimentos que seriam oferecidos. Caso não, este era trocado por outro semelhante. Esta mudança ocorreu somente para um participante para o qual a bolacha salgada foi substituída por bolacha doce. Assim que chegava à sala de coleta, era dito à criança que ela faria um lanche e era solicitado que se sentasse na cadeira bem próxima à mesa. A pesquisadora se posicionava atrás do participante de forma a não ser vista por ele (evitar pistas visuais) e simultaneamente colocava duas embalagens transparentes idênticas sobre a mesa. Estas embalagens continham amostras do mesmo alimento, tendo somente como distintos os rótulos na tampa. Os alimentos utilizados foram bolacha salgada (com exceção de um participante) no Teste 1 , suco de uva (Teste 2) e chocolate (Teste 3). No Teste de escolha era dada a seguinte instrução: "Aqui estão dois (dizia-se o nome do alimento). Você poderá comer (ou beber) os dois. Qual deles você quer comer (ou beber) primeiro?" O participante escolhia o alimento e esperava-se até que ele terminasse de consumi-lo. No teste de preferência, era solicitado que comesse ou bebesse o outro também e perguntava-se qual dos dois ela havia gostado mais. Caso a criança não demonstrasse preferência, alegando, por exemplo, que os alimentos eram iguais, era "forçada" uma resposta: "Tem certeza? Você deve ter gostado mais de um". Os três testes de escolha e preferência eram realizados na sequência um do outro, seguindo a mesma ordem para todos os participantes.

No Teste 1 a escolha era entre o alimento com o rótulo contendo o símbolo da classe do personagem "positivo" (C1) e o outro do "negativo" (C2). No Teste 2 a escolha era entre o símbolo C2 e um símbolo novo (SN1). No Teste 3 o participante deveria escolher entre o $\mathrm{C} 1$ e um outro símbolo novo (SN2). A posição dos rótulos das embalagens (direita ou esquerda) variava entre os testes.

\section{Fase 4 - Teste de manutenção das classes e de transferência de função}

Passadas duas semanas, os testes de equivalência (AC-CA) eram conduzidos novamente para verificar a manutenção das classes de estímulos. $\mathrm{Na}$ sequência, repetiam-se os testes de escolha e preferência de alimentos, seguindo os mesmos procedimentos detalhados anteriormente.

\section{Resultados}

Os resultados do treino e teste relacional para cada participante são apresentados na Tabela 1 . Todos os participantes concluíram as fases de treino e teste, sendo que a maioria não necessitou mais do que um bloco de tentativas para atingir o critério, tanto na fase de treino quanto na de teste. Todos eles passaram no teste de equivalência, com exceção do P10 que passou no teste de transitividade (AC), mas não atingiu o critério no teste $\mathrm{CA}$. Na fase de 
manutenção, realizada duas semanas depois, todos os participantes demonstraram a formação das classes. P8 não atingiu o critério no teste $\mathrm{AC}$, mas passou no teste de equivalência CA. P10, que não havia atingido o critério no primeiro teste, apresentou desempenho esperado na fase de manutenção.

A Tabela 2 apresenta os resultados dos testes de escolha e preferência de cada participante feitos após o teste de equivalência (Pós-teste). P2 foi excluído dos resultados por possuir restrição alimentar (não estava habituado a comer alimentos industrializados). Como se pode observar no Teste 1, realizado no Pós-teste, dos dez participantes, 90\% escolheu provar primeiro e também demonstrou preferência pelo alimento com o rótulo C1 (equivalente ao personagem de que gostavam). $\mathrm{O}$ teste chi quadrado apontou que a diferença entre estas escolhas foi significativa $\left(\chi^{2}(1)=6,400 ; p<0,011\right)$. Somente P1 escolheu e demonstrou preferência pelo alimento com o rótulo C2. Foi necessário "forçar” uma resposta para os participantes P1, P3, P6 e P9, pois alegaram a princípio, que os alimentos eram iguais. Com relação ao Teste 2, 60\% dos participantes escolheram provar primeiro o alimento com o rótulo $\mathrm{C} 2$ (equivalente ao personagem de que não gostavam) e os demais (40\%) escolheram o símbolo novo ( $\mathrm{SN} 1)$, não sendo esta diferença significativa $\left(\chi^{2}(1)=0,400 ; p<0,527\right)$. Com relação à preferência, somente 4 dos que escolheram provar primeiro o alimento com o rótulo $\mathrm{C} 2$, mantiveram a preferência por ele. O restante $(60 \%)$ disse ter gostado mais do alimento com o símbolo novo (SN1). No Teste 3, 60\% dos participantes escolheram provar primeiro o alimento com o rótulo $\mathrm{C} 1$ e o restante escolheu o alimento com um símbolo novo (SN2). Novamente esta diferença não foi significativa $\left(\chi^{2}(1)=0,400 ; p<0,527\right)$. Quando perguntados qual havia gostado mais, somente dois participantes que escolheram provar primeiro o alimento com $\mathrm{SN} 2$ no rótulo mantiveram a preferência por ele. $70 \%$ dos participantes alegaram ter gostado mais do alimento com o rótulo $\mathrm{C} 1\left(\chi^{2}(1)=1,600 ; p<0,206\right)$.

Tabela 1. Idade, Gênero e Número de Tentativos Corretas por Blocos nas Fases do Treino e Teste Relacional de cada Participante

\begin{tabular}{|c|c|c|c|c|c|c|c|c|c|c|}
\hline P\# & Sexo & Idade & $A B$ & BA & $\mathrm{BC}$ & CB & $A C_{1}$ & CA 1 & AC 2 & CA 2 \\
\hline $\mathrm{P} 1$ & $\mathrm{~F}$ & 5,9 & $9 / 12 ; 10 / 10$ & $8 / 8$ & $12 / 12$ & $7 / 8$ & $7 / 8$ & $7 / 8$ & $7 / 8$ & $8 / 8$ \\
\hline $\mathrm{P} 2$ & $M$ & 5,2 & $12 / 12$ & $8 / 8$ & $11 / 12$ & $7 / 8$ & $7 / 8$ & $7 / 8$ & $8 / 8$ & $6 / 8 ; 7 / 8$ \\
\hline P3 & M & 5,5 & $12 / 12$ & $8 / 8$ & $12 / 12$ & $8 / 8$ & $8 / 8$ & $8 / 8$ & $8 / 8$ & $8 / 8$ \\
\hline $\mathrm{P} 4$ & $\mathrm{~F}$ & 4,11 & $11 / 12$ & $8 / 8$ & $11 / 12$ & $8 / 8$ & $8 / 8$ & $8 / 8$ & $8 / 8$ & $7 / 8$ \\
\hline P5 & $M$ & 5,5 & $11 / 12$ & $8 / 8$ & $11 / 12$ & $8 / 8$ & $8 / 8$ & $7 / 8$ & $8 / 8$ & $7 / 8$ \\
\hline P6 & $\mathrm{F}$ & 5,7 & $7 / 12 ; 10 / 10$ & $7 / 8$ & $12 / 12$ & $8 / 8$ & $8 / 8$ & $8 / 8$ & $8 / 8$ & $7 / 8$ \\
\hline $\mathrm{P} 7$ & $M$ & 4,8 & $12 / 12$ & $8 / 8$ & $12 / 12$ & $8 / 8$ & $8 / 8$ & $8 / 8$ & $8 / 8$ & $7 / 8$ \\
\hline P8 & $\mathrm{F}$ & 5,5 & $11 / 12$ & $7 / 8$ & $\begin{array}{l}10 / 12 \\
10 / 10\end{array}$ & $7 / 8$ & $8 / 8$ & $2 / 8 ; 8 / 8$ & $5 / 8 ; 6 / 8$ & $8 / 8$ \\
\hline P9 & $M$ & 5,6 & $\begin{array}{c}9 / 12 ; 6 / 10 \\
10 / 10\end{array}$ & $8 / 8$ & $11 / 12$ & $8 / 8$ & $8 / 8$ & $8 / 8$ & $8 / 8$ & $7 / 8$ \\
\hline P10 & M & 5,0 & $11 / 12$ & $\begin{array}{l}4 / 8 ; \\
7 / 8\end{array}$ & $\begin{array}{c}10 / 12 ; 8 / 10 \\
10 / 10\end{array}$ & $8 / 8$ & $8 / 8$ & $6 / 8 ; 4 / 8$ & $8 / 8$ & $8 / 8$ \\
\hline P11 & $M$ & 5,6 & $11 / 12$ & $7 / 8$ & $10 / 12 ; 9 / 10$ & $\begin{array}{l}6 / 8 \\
8 / 8\end{array}$ & $8 / 8$ & $7 / 8$ & $8 / 8$ & $8 / 8$ \\
\hline
\end{tabular}

Nota. As colunas marcadas (AC2 e CA2) correspondem ao Teste de Manutenção. 
Tabela 2. Desempenho de cada participante nos testes de escolha e preferência de alimentos na Fase Pós-teste

\begin{tabular}{c|c|c|c|c|c|c}
\hline \multirow{2}{*}{ P\# } & \multicolumn{2}{|c|}{$\begin{array}{c}\text { Teste 1 } \\
\text { C1 vs C2 }\end{array}$} & \multicolumn{2}{c|}{$\begin{array}{c}\text { Teste 2 } \\
\text { C2 vs SN1 }\end{array}$} & \multicolumn{2}{c}{$\begin{array}{c}\text { Teste 3 } \\
\text { SN2 vs C1 }\end{array}$} \\
\hline & E & P & E & P & E & P \\
\hline P1 & C2 & NP/C2 & C2 & SN1 & C1 & C1 \\
\hline P3 & C1 & NP/C1 & SN1 & SN1 & C1 & C1 \\
\hline P4 & C1 & C1 & SN1 & SN1 & C1 & SN2 \\
\hline P5 & C1 & C1 & C2 & C2 & C1 & C1 \\
\hline P6 & C1 & NP/C1 & C2 & C2 & C1 & C1 \\
\hline P7 & C1 & C1 & SN1 & SN1 & SN2 & C1 \\
\hline P8 & C1 & C1 & C2 & C2 & SN2 & C1 \\
\hline P9 & C1 & NP/C1 & C2 & SN1 & SN2 & SN2 \\
\hline P10 & C1 & C1 & SN1 & SN1 & SN2 & SN2 \\
\hline P11 & C1 & C1 & C2 & C2 & C1 & C1 \\
\hline
\end{tabular}

Nota: E: Escolha; P: Preferência; NP: Não Preferência; SN1: Símbolo Novo 1; SN2: Símbolo Novo 2.

Os testes realizados na Fase de Manutenção (Tabela 3) seguiram a mesma tendência de escolha e preferência observada no Pós-teste. No Teste 1 , $80 \%$ dos participantes escolheram primeiro o alimento com o rótulo $\mathrm{C} 1\left(\chi^{2}(1)=6,300 ; p<0,058\right)$. Destes, $70 \%$ mantiveram a preferência por ele $\left(\chi^{2}\right.$ $(1)=1,600 ; p<0,206)$. Comparando com o resultado do Pós-teste, os participantes P1, P6 e P9 modificaram tanto a escolha quanto a preferência. É importante destacar que estes mesmos participantes haviam indicado não preferência na fase anterior. P7 modificou somente a preferência. No Teste 2 da Fase de Manutenção a maioria dos participantes (70\%) escolheu o alimento com o símbolo novo (SN1) no rótulo ao invés do alimento com o rótulo $\mathrm{C} 2\left(\chi^{2}(1)=1,600 ; p<0,206\right)$, ou seja, a escolha do SN1 foi superior na Fase de Manutenção do que na Fase Pós-teste. Como se pode observar, três participantes (P1, P6 e P11) mudaram suas respostas tanto em relação à escolha quanto à preferência, comparado à fase anterior. No entanto, destes sete participantes, somente cinco mantiveram a preferência pelo que haviam escolhido pri- meiro, e o restante disse ter gostado mais do alimento com o rótulo C2. Além dos já citados, o P8 e P9 modificaram somente suas preferências. No Teste 3, $70 \%$ dos participantes escolheram provar primeiro o alimento com rótulo $\mathrm{C} 1\left(\chi^{2}(1)=1,600\right.$; $p<0,206)$. Somente três participantes modificaram suas escolhas em relação à Fase Pós-teste (P1, P8 e P9). Já em relação à preferência, seis deles disseram que preferiram o mesmo alimento. P4, P5 e P7 somente modificaram suas preferências comparadas à fase do Pós-teste.

Considerando uma análise mais geral dos resultados da Fase de Manutenção, é interessante notar que, somente três participantes de cada teste específico mudaram suas escolhas (Teste 1: P1, P6 e P9; Teste 2: P1, P6 e P11; Teste 3: P1, P8 e P9). Como se percebe, destes participantes, P1, P6 e P9 mudaram suas escolhas em mais de um teste. P1, por exemplo, alterou suas respostas no Teste 1, 2 e 3 . Com relação à preferência, houve maior variabilidade nas respostas, principalmente considerando os Testes 2 e 3 desta fase. 
Tabela 3. Desempenho de cada participante nos testes de escolha e preferência de alimentos na Fase de Manutenção

\begin{tabular}{c|c|c|c|c|c|c}
\hline P\# & \multicolumn{2}{|c|}{$\begin{array}{c}\text { Teste 1 } \\
\text { C1 vs C2 }\end{array}$} & \multicolumn{2}{c|}{$\begin{array}{c}\text { Teste 2 } \\
\text { C2 vs SN1 }\end{array}$} & \multicolumn{2}{c}{$\begin{array}{c}\text { Teste 3 } \\
\text { C1 vs SN2 }\end{array}$} \\
\hline & E & P & E & P & E & P \\
\hline P1 & C1 & C1 & SN1 & C2 & SN2 & C1 \\
\hline P3 & C1 & C1 & SN1 & SN1 & C1 & C1 \\
\hline P4 & C1 & C1 & SN1 & SN1 & C1 & C1 \\
\hline P5 & C1 & C1 & C2 & C2 & C1 & SN2 \\
\hline P6 & C2 & C2 & SN1 & SN1 & C1 & C1 \\
\hline P7 & C1 & NP/C2 & SN1 & C2 & SN2 & SN2 \\
\hline P8 & C1 & C1 & C2 & SN1 & C1 & C1 \\
\hline P9 & C2 & C2 & C2 & C2 & C1 & C1 \\
\hline P10 & C1 & C1 & SN1 & SN1 & SN2 & SN2 \\
\hline P11 & C1 & C1 & SN1 & SN1 & C1 & C1 \\
\hline
\end{tabular}

Nota: E: Escolha; P: Preferência; NP: Não Preferência; SN1: Símbolo Novo 1; SN2: Símbolo Novo 2.

As linhas marcadas correspondem aos participantes que mudaram suas respostas em relação ao Pós-teste.

\section{Discussão}

O presente estudo teve como objetivo investigar se o significado de figuras de personagens infantis, supostamente positivas e negativas seria transferido para símbolos abstratos, por meio de relações derivadas, e estes influenciariam escolhas e preferências alimentares de crianças, quando dispostos em rótulos de embalagens. Os resultados mostraram que, mesmo os alimentos sendo idênticos, quando a escolha envolve um símbolo equivalente a um personagem de que a criança gosta e o outro equivalente ao que não gosta, as crianças tendem a escolher e preferir o primeiro. Os dados obtidos corroboram os resultados de pesquisas anteriores que adotaram uma metodologia semelhante (Arntzen et al., 2016; Santos \& de Rose (no prelo), Smeets \& BarnesHolmes, 2003; Barnes-Holmes et al., 2000).

Os resultados também demonstraram que, após duas semanas, os participantes ainda mantinham a formação das classes de estímulos equivalentes, conforme tem sido destacado na literatura (Aggio \& Domeniconi, 2012; Almeida \& Haydu, 2011; Rehfeldt \& Dixon, 2005). A maior contribuição foi a estabilidade da transferência de função observada nos testes de escolha e preferência da Fase de Manutenção, confirmando assim a primeira hipótese deste estudo. Há escassez de trabalhos na literatura sobre este tema, no entanto, a pesquisa de Silveira et al. (2016) também apontou resultados favoráveis de manutenção da transferência de função. Naquela pesquisa, feita com universitários, eles utilizaram o diferencial semântico (conforme proposto por Bortoloti \& de Rose, 2007, 2009) para medir a transferência de significado entre expressões faciais de alegria, neutralidade e raiva e figuras abstratas. Trinta dias depois, os participantes que mantiveram o desempenho no teste de equivalência também continuaram demonstrando transferência de função, tendo um efeito mais forte para a classe de significado positivo (face alegre). A presente pesquisa contribui no sentido de corroborar estes resultados de manutenção de classes e transferência de função utilizando, possivelmente de forma pioneira, uma população infantil.

As outras hipóteses do estudo, referentes à transferência de função negativa no Teste 2 , e de função positiva no Teste 3, não foram confirmadas. A inserção de símbolos novos nos testes parece ter gerado maior variabilidade nas respostas. Os resultados encontrados nos Testes 2 e 3 não foram estatisticamente significativos, ou seja, não foi possível afirmar qual dos estímulos teve maior efeito sobre as respostas. No entanto, na Fase de Manutenção, os resultados foram mais condizentes com as hipóteses, ou seja, houve prevalência de escolha pelo SN1 no Teste 2 e pelo $\mathrm{C} 1$ no Teste 3 . Um argumento possível seria a emergência atrasada destas relações, 
no entanto, deve ser considerado também o provável efeito da história de aprendizagem gerado a partir das próprias contingências dos testes. A criança, por exemplo, pode ter escolhido provar um alimento com um rótulo diferente, não por preferência, mas por não tê-lo experimentado antes. Este efeito pode ser ainda maior no Teste de Manutenção. Pesquisas futuras devem tentar controlar estas variáveis, mudando a ordem de realização dos testes ou trabalhando com diferentes grupos experimentais.

No estudo de Santos e de Rose (no prelo) um percentual maior de crianças escolheu o alimento com símbolo novo na embalagem ao invés do símbolo equivalente ao personagem de que não gostava, indicando uma possível rejeição devido à transferência de função negativa. No entanto, não foi possível confirmar estas hipóteses no presente estudo, o que reforça que a novidade do estímulo pode ter um efeito importante nestas escolhas (Grisante, de Rose, \& McIIvane, 2014; Valenti, 1985).

Independente destas questões, o presente estudo e pesquisas anteriores (Arntzen et al., 2016; BarnesHolmes et al., 2000; Santos e de Rose [submetido], Smeets, \& Barnes-Holmes, 2003) demonstram que a equivalência de estímulos pode ser um paradigma útil para investigar a formação de atitudes positivas em relação a marcas que utilizam personagens atrativos em suas estratégias. Normalmente, o condicionamento clássico tem sido adotado como modelo de explicação sobre como estímulos adquirem funções emocionais e interferem nas escolhas de produtos (Macklin, 1986; Stuart, Shimp, \& Engle, 1987). Este processo também tem sido conhecido como Evaluative Conditioning (EC) (para uma revisão ver De Houwer, Thomas, \& Baeyens, 2001). De maneira geral, o EC se refere à mudança na valência de um estímulo considerado neutro devido ao seu pareamento sistemático a outro estímulo que pode ser positivo ou negativo. Entretanto, dados têm demonstrado que os efeitos deste tipo de condicionamento são breves e elusivos (e.g. de Howver, Baeyens, \& Field, 2005; Jones, Olson, \& Fazio, 2010).

$\mathrm{Na}$ presente pesquisa, personagens e símbolos abstratos não foram pareados diretamente de forma sistemática e, além disto, no matching atrasado, o estímulo modelo sempre é removido antes da apresentação dos estímulos de comparação. Assim, os resultados obtidos não poderiam ser atribuídos a um efeito de condicionamento avaliativo. Por outro lado, estudos sobre transferência de função têm demonstrado várias evidências de que estímulos equivalentes se tornam substituíveis uns aos outros, podendo evocar diferentes tipos de respostas, inclusive emocionais (e.g., Amd, Barnes-Holmes, \&, 2013; Bortoloti \& de Rose, 2009, 2012; de Almeida \& de Rose, 2015; de Rose et al., 1988; Dougher et al., 1994; Wulfert \& Hayes, 1988). Pesquisas sobre medo e ansiedade, por exemplo, têm mostrado que o medo evocado por um membro da classe pode se transferir para outros membros (e.g., Bennett, Meulders, Baeyens, \&Vlaeyen, 2015; Dymond, Dunsmoor, Vervliet, Roche, \& Hermans, 2015), um efeito também chamado de generalização simbólica ou baseado em categorias.

As pesquisas na área de marketing apontam que as crianças tendem a preferir alimentos que possuem personagens ou mascote em suas embalagens (Elliott, Hoed, \& Conlon, 2013; Kotler, Schiffman, \& Hanson, 2012; Lapierre, Vaala \& Linebarger, 2011; Roberto, Baik, Harris, \& Brownell, 2010). Além disto, crianças pequenas avaliam o mesmo produto como mais gostoso somente por estar em uma embalagem com uma marca conhecida (Robinson, Borzekowski, Matheson \& Kraemer, 2007).

No caso da presente pesquisa, símbolos abstratos que, a princípio não teriam nenhum significado, passaram a exercer o mesmo controle que os personagens exerceriam sobre a resposta da criança, interferindo em sua atitude ou preferência em relação aos alimentos. Assim, os resultados obtidos sugerem que somente um símbolo que se tornou equivalente ao personagem na embalagem é capaz de controlar a mesma resposta através da transferência de função. Estas constatações reforçam o poder de persuasão que as propagandas exercem sobre as escolhas alimentares de crianças (para uma revisão ver Cairns, Angus, Hastings, \& Caraher, 2013; Kraak \& Story, 2015). Considerando o aumento da obesidade infantil em todo o mundo (WHO, 2015), os dados encontrados se mostram de grande relevância social, pois reforçam a necessidade de discussão sobre as questões éticas que envolvem as propagandas e suas formas de regulamentação.

Concluindo, os resultados desta pesquisa apontam que a escolha do alimento com símbolo no ró- 
tulo equivalente ao personagem que a criança gosta tende a prevalecer, quando comparado ao que ela não gosta, e tais escolhas parecem se manter ao longo da tempo. Além disto, outro aspecto importante destes resultados foi que as respostas dos participantes não sofreram interferência com relação ao balanceamento dos estímulos $\mathrm{C} 1$ e $\mathrm{C} 2$ entre as classes positiva e negativa. Isto indica que o desempenho obtido ocorreu por meio da transferência de significado entre personagens e símbolos abstratos e não devido a uma possível preferência às características destes símbolos.

Uma possível limitação deste estudo se refere ao método de escolha dos estímulos significativos (personagens). O uso de avaliação de preferência pareada não se mostrou ser uma alternativa eficiente para esta população, à medida que a maioria dos participantes demonstrou desinteresse pela tarefa, provavelmente devido à repetição de itens. Outro fator que pode ter interferido é o fato da figura adotada como "negativa" ser um personagem de desenho e, independentemente de ser avaliado como agradável ou não à criança, faz parte do contexto infantil. Sugere-se que pesquisas futuras adotem outras estratégias para escolhas destes estímulos, bem como utilizem medidas de significado, tendo em vista ser esta uma variável importante para a investigação da transferência de função (Bortoloti, Rodrigues, Cortez, Pimentel, \& de Rose, 2013; Silveira et al., 2016).

\section{Referências}

Amd, M., Barnes-Holmes, D., \& Ivanoff, J. (2013). A derived transfer of eliciting emotional functions using differences among electroencephalograms as a dependent measure. Journal of the Experimental Analysis of Behavior, 99, 318-334. doi: $10.1002 /$ jeab. 19

Adams, B. J., Fields, L., \& Verhave, T. (1993). Effects of test order on intersubject variability during equivalence class formation. The Psychological Record, 43, 133-152.

Aggio, N. M., \& Domeniconi, C. (2012). Formação e manutenção de classes de estímulos equivalentes: um estudo com participantes da terceira idade. Acta Comportamentalia, 20, 29-43.
Almeida, J. H., \& Haydu, V. B. (2011). Reorganization of equivalence classes: analysis of reversed baseline relations. Psicologia Reflexão e Crítica, 24, 609-620. doi:10.1590/ S0102-79722011000300022.

Arntzen, E., Fagerstrom, A., \& Foxall, G. (2016). Equivalence classes and preferences in consumer choices. In Gordan R. Foxall (Ed.), The Routledge Companion to Consumer Behavior Analysis (pp. 65-77). Publisher: Routledge.

Barnes-Holmes, D., Keane, J., Barnes-Holmes, Y. \& Smeets, P. M. (2000). A derived transfer of emotive functions as a means of establishing differential preferences for soft drinks. The Psychological Record, 50, 493-511.

Barnes-Holmes, Y., Barnes-Holmes, D., Smeets, P. M., \& Luciano, C. (2004). A derived transfer of mood functions through equivalence relations. The Psychological Record, 54, 95-104.

Barnes-Holmes, D., Staunton, C., Whelan, R., Barnes-Holmes, Y., Commins, S., Walsh, D., \& Dymond, S. (2005). Derived stimulus relations, semantic priming, and event-related potentials: testing a behavioral theory of semantic networks. Journal of the Experimental Analysis of Behavior, 84, 417-433.doi: 10.1901/ jeab.2005.78-04.

Bennett, M. P., Meulders, A., Baeyens, F., \& Vlaeyen, J. W. S. (2015). Words putting pain in motion: the generalization of pain-related fear within an artificial stimulus category. Frontiers in Psychology, 6, 20. doi:10.3389/fpsyg.2015.00520

Bortoloti, R., \& de Rose, J. C. (2007). Medida do grau de relacionamento entre estímulos equivalentes [Measuring the degree of relatedness between equivalent stimuli]. Psicologia: Reflexão e Crítica, 20 (2), 250-256.

Bortoloti, R., \& de Rose, J. C. (2009) Assessment of the relatedness of equivalent stimuli through a semantic differential. The Psychological Record, 59, 534-590.

Bortoloti, R., \& de Rose, J. C. (2011). Avaliação do efeito de dica semântica e da indução de significado entre estímulos abstratos equivalentes [Assessment of the effects of semantic priming and induction of meaning between abstract equivalent stimuli]. Psicologia: Reflexão e Crítica, 24 (2), 381-393. 
Bortoloti, R., \& de Rose, J. C. (2012). Equivalent stimuli are more strongly related after training with delayed matching than after simultaneous matching: A study using the implicit relational assessment procedure (IRAP). The Psychological Record, 62 (1), 41-54.

Bortoloti, R., Rodrigues, N. C., Cortez, M. D., Pimentel, N., \& de Rose, J. C. (2013). Overtraining increases the strength of equivalence relations. Psychology \& Neuroscience, 6, 357-364. doi: 10.3922/j.psns.2013.3.13

Bortoloti, R., Pimentel, N., \& de Rose, J. C (2014). Electrophysiological investigation of the functional overlap between semantic and equivalence relations. Psychology \& Neuroscience, 7(2), 183-191. https://dx.doi.org/10.3922/j. psns.2014.015

Cairns, G., Angus, K., Hastings, G., \& Caraher, M. (2013). Systematic reviews of the evidence on the nature, extent and effects of food marketing to children. A retrospective summary. Appetite, 62, 209-215.

de Almeida, J. H., \& de Rose, J. C. (2015). Changing the Meaningfulness of Abstract Stimuli by the Reorganization of Equivalence Classes: Effects of Delayed Matching, The Psychological Record, 65, 451-461. doi: 10.1007/s40732-015-0120-9.

de Carvalho, M. P., \& de Rose, J. C. (2014). Understanding racial attitudes through the stimulus equivalence paradigm. The Psychological Record, 64, 527-536. doi: 10.1007/s40732-0140049-4

De Houwer, J., Thomas, S., \& Baeyens, F. (2001). Associative learning of likes and dislikes: a review of 25 years of research on human evaluative conditioning. Psychological Bulletin, 127, 853-869. doi: 10.1037/0033-2909.127.6.853.

De Houwer, J., Baeyens, F., \& Field, A. P. (2005). Associative learning of likes and dislikes: Some current controversies and possible ways forward. Cognition and Emotion, 19, 161-174.

de Rose, J. C., McIlvane, W. J., Dube, W. V., Galpin, V. C., \& Stoddard, L. T. (1988). Emergent simple discriminations established by indirect relations to differential consequences. Journal of the Experimental Analysis of Behavior, 50, 1-20.doi: 10.1901/jeab.1988.50-1
Dougher, M. J., Augustson, E., Markham, M. R., Greenway, D. E., \& Wulfert, E. (1994). The transfer of respondent eliciting and extinction functions through stimulus equivalence classes. Journal of the Experimental Analysis of Behavior, 62 (3), 331-351.doi: 10.1901/jeab.1994.62-331

Dymond, S., Dunsmoor, J. E., Vervliet, B., Roche, B., \& Hermans, D. (2015). Fear Generalization in Humans: Systematic Review and Implications for Anxiety Disorder Research. Behavior Therapy, 45, 561-582.doi.org/10.1016/j. beth.2014.10.001.

Elias, N. C., \& Goyos, C. (2010). Mestre Libras no Ensino de Sinais: Tarefas Informatizadas de Escolha de Acordo com o Modelo e Equivalência de Estímulos. In E. G. Mendes and M. A. Almeida. (Eds.). Das Margens Ao Centro: Perspectivas para as Políticas e Práticas Educacionais no Contexto da Educação Especial Inclusiva, pp. 223-234). Araraquara: Junqueira \& Marin Editora e Comercial Ltda.

Elliot, C. D., Den Hoed, R. C., \& Conlon, M. J. (2013). Food Branding and Young Children's Taste Preferences: A Reassessment. Canadian Journal of Public health, 104 (5), 364-368.

Escobal, G., Elias, N. C., \& Goyos, C. (2012). Jogo da Escolha: Ferramenta informatizada para avaliar preferências por reforçadores. Temas em Psicologia, 20 (2), 451-458. doi:10.9788/ TP2012.2-13

Escobal, G., Elias, N. C., \& Goyos, C. (2014). Comparação entre Avaliações de Preferência com Itens Tangíveis e com Itens Digitais. Temas em Psicologia, 22 (1), 235-248. doi: 10.9788/ TP2014.1-18

Fisher, W. W., Piazza, C. C., Bowman, L. G., Hagopian, L. P., Owens, J. C., \& Slevin, I. (1992). A comparison of two approaches for identifying reinforcers for persons with severe and profound disabilities. Journal of Applied Behavior Analysis, 25, 491-498.

Grey, I. M., \& Barnes, D. (1996). Stimulus Equivalence and Attitudes. The Psychological Record, 43, 243-270.

Grisante, P. C., Rose, J. C. de, \& McIlvane, W. J. (2014). Controlling relations in stimulus equivalence classes of preschool children and individuals with Down Syndrome. The Psychological 
Record, 64(2), 195-208. doi:10.1007/s40732014-0021-3

Haimson, B., Wilkinson, K. M., Rosenquist, C., Ouimet, C., \& McIlvane, W. J. (2009). Electrophysiological correlates of stimulus equivalence processes. Journal of the Experimental Analysis of Behavior, 92(2), 245256.doi: 10.1901/jeab.2009.92-245.

Haydu, V. B., Camargo, J., \& Bayer, H. (2015). Effects of preexperimental history on the formation of stimulus equivalence classes: A study with supporters of Brazilian soccer clubs. Psychology \& Neuroscience, 8(3), 385-396.

Jones, C. R., Olson, M. A., \& Fazio, R. H. (2010). Evaluative conditioning: The "how" question. Advances in Experimental Social Psychology, 1(43), 205-255.

Kotler, J. A., Schiffman, J. M., \& Hanson, K. G. (2012). The Influence of media characters on children's food choices. Journal of Health Communication, 17(8), 886-898. doi: 10.1080/10810730.2011.650822

Kraak, V. I., \& Story, M. (2015). Influence of food companies' brand mascots and entertainment companies' cartoon media characters on children's diet and health: a systematic review and research needs. Obesity Reviews, 16, 107126. doi: 10.1111/obr.12237.

Lapierre, M. A., Vaala, S. E., \& Linebarger, D. L. (2011). Influence of licensed spokes characters and health cues on children's ratings of cereal taste. Archives of Pediatrics \& Adolescent Medicine, 165 (3), 229-234.

Macklin, N. C. (1986). Classical conditioning effects in product/character pairings presented to children. Advances in Consumer Research, 13, 198-203.

Mizael, T. M., de Almeida, J. H., Silveira, C. C., \& de Rose, J. C. (2016). Changing racial bias by transfer of functions in equivalence classes. The Psychological Record, 66, 451-462. doi: 10.1007/ s40732-016-0185-0

Mizael, T. M., Santos, S. L., \& de Rose, J. C. (2016). Contribuições da equivalência de estímulos para o estudo das atitudes. Interação em Psicologia. 20, 2, 124-134. doi: http://dx.doi. org/10.5380/psi.v20i2.46278
Moxon, P., Keenan, M., \& Hine, L. (1993). Genderrole stereotyping and stimulus equivalence. The Psychological Record, 43, 381-394.

Rehfeldt, R. A., \& Dymond, S. (2005). The effects of test order and nodal distance on the emergence and maintenance of derived discriminative stimulus functions. The Psychological Record, 55, 179-196.

Roberto, C. A., Baik, J., Harris, J. L., \& Brownell, K. D. (2010). Influence of Licensed characters on Children's Taste and Snack Preferences. Pediatrics, 26, 88-93.

Robinson, T. N., Borzekowski, D. L. G., Matheson, D. M, \& Kraemer, H. C. (2007). Effects of Fast Food Branding on Young Children's Taste Preferences. Archives of Pediatrics \& Adolescent Medicine, 161(8), 792-797.

Santos, S. L., \& de Rose, J. C. (no prelo). Investigating the Impact of Stimulus Equivalence on Children's Food Choice and Preference. Trends in Psychology.

Sidman, M., \& Tailby, W. (1982) Conditional discrimination vs. matching to sample: an expansion of the testing paradigm. Journal of the Experimental Analysis of Behavior, 37 (1), 5-22, 1982. doi: 10.1901/jeab.1982.37-5

Sidman, M. (1994) Equivalence Relations and Behavior. A Research Story. Boston: Authors Cooperative.

Sidman, M. (2000). Equivalence relations and the reinforcement contingency. Journal of the Experimental Analysis of Behavior, 74, 127-146.

Silveira, J. H., Aggio, N. M., Cortez. M. D., Bortoloti, R., Rico, V. V., \& de Rose, J. C. (2016). Maintenance of Equivalence Classes and Transfer of Functions: The Role of the Nature of Stimuli. The psychological Record, 66, 65-74. doi: 10.1007/s40732-015-0152-1.

Smeets, P. M., \& Barnes-Holmes, D. (2003). Children's emergent preferences for soft drinks: Simulus-equivalence and transfer. Journal of Economic Psychology, 24, 603-618.

Straatmann, G., Almeida, S. S., \& de Rose, J. C. (2014).Computerized Assessment of Food Preferences in Adolescents in the Stimulus Equivalence Paradigm. Trends in Psychology, 22(3), 613-624.doi: 10.9788/TP2014.3-07 
Stuart, E. W., Shimp, T. A., \& Engle, R. W. (1987). Classical Conditioning of Consumer Attitudes: Four Experiments in an Advertising Context. Journal of Consumer Research, 14, 334-49

Valenti, S. S. (1985) Children's preference for novelty in selective learning: Developmental stability or change? Journal of the Experimental Child Psychology, 40, 406-419.

Watt, A., Keenan, M., Barnes, D., \& Cairns, E. (1991). Social categorization and stimulus equivalence. The Psychological Record, 41, 33-50

World Health Organization (2015). Childhood overweight and obesity. Retrieved from http:// www.who.int/dietphysicalactivity/childhood/en/

Wulfert, E., \& Hayes, S. C. (1988). Transfer of a conditional ordering response through conditional equivalence classes. Journal of the Experimental Analysis of Behavior, 50, 125-144.doi: 10.1901/ jeab.1988.50-125

Informações do Artigo

Histórico do artigo:

Submetido em: 16/06/2016

Aceito em: 06/12/2016

Editor Associado: Saulo Velasco 